\title{
Probiotic and glutamine treatments attenuate alcoholic liver disease in a rat model
}

\author{
HUPING HUANG ${ }^{1-3 *}$, ZHIHUI LIN ${ }^{2-3 *}$, YANLING ZENG ${ }^{2,3}$, XUEYAN LIN $^{2,3}$ and YALI ZHANG ${ }^{1}$ \\ ${ }^{1}$ Department of Gastroenterology, Nanfang Hospital, Southern Medical University, Guangzhou, Guangdong 510515; \\ ${ }^{2}$ Department of Gastroenterology, Fujian Provincial Hospital, Fujian Medical University; \\ ${ }^{3}$ Fujian Institute of Gastroenterology, Fuzhou, Fujian 350000, P.R. China
}

Received January 7, 2019; Accepted August 17, 2019

DOI: $10.3892 /$ etm.2019.8123

\begin{abstract}
The pathogenesis underlying alcoholic liver disease (ALD), which is often a result of alcohol abuse, currently remains unclear. Previous studies have reported that enteric dysbiosis serves an important role in the pathogenesis of ALD. The present study aimed to investigate the effects of glutamine and probiotics on a rat model of alcoholic liver disease (ALD). Sixty male Sprague-Dawley rats were randomly divided into 6 groups including control (C), alcohol (M), alcohol + Golden Bifido $(\mathrm{T})$, alcohol + glutamine $(\mathrm{G})$, alcohol + Medilac-S ${ }^{\circledR}$ (N) and alcohol + Golden Bifido + glutamine (L). Histology, body weight (BW), triglycerides (TG), serum aspartate transaminase (AST), alanine aminotransferase (ALT), tumor necrosis factor (TNF- $\alpha$ ), interleukin-6 (IL-6), diamine oxidase (DAO), occludin, endotoxin and D-lactate levels were assessed whilst changes in the gut flora were evaluated and compared. Results determined that all probiotic and glutamine treatments elevated the abnormally decreased BW and occludin levels whilst the abnormal elevated serum AST, ALT, TG, IL-6, TNF- $\alpha$, DAO, endotoxin and D-lactate levels were significantly reduced following chronic ethanol consumption. Histopathological observation of the liver demonstrated that probiotic and glutamine treatments attenuated liver damage induced by alcohol. Moreover, sequencing determined that there was a reduction in Firmicutes as well as an increase in Actinobacteria, Proteobacteria and Porphyromonadaceae abundance in the ALD group compared with the healthy controls. However, these changes were prevented by glutamine and probiotic therapy. In conclusion, the present results
\end{abstract}

Correspondence to: Dr Yali Zhang, Department of Gastroenterology, Nanfang Hospital, Southern Medical University, 1838 North Guangzhuou Avenue, Guangzhou, Guangdong 510515, P.R. China

E-mail: zyl41531@163.com

${ }^{*}$ Contributed equally

Key words: alcoholic liver disease, probiotics, glutamine, gut microbiota suggested that probiotics and glutamine ameliorated ALD by suppressing inflammation and regulating the gut microbiota. Therefore, probiotic and glutamine treatments can potentially serve as therapies for the prevention and treatment of ALD.

\section{Introduction}

Alcoholic liver disease (ALD) is a major cause of morbidity and mortality worldwide amongst people who abuse alcohol (1). The spectrum of ALD ranges from simple steatosis to alcoholic hepatitis, fibrosis, cirrhosis and ultimately hepatocarcinoma (2). To date, few satisfactory advances have been made in the management of ALD. Thus, novel and more reliable therapeutic approaches are urgently required.

Several factors have been demonstrated to contribute to the progression of ALD in humans, including race, gender, ethnicity and comorbidities such as hepatitis $\mathrm{B}$ and $\mathrm{C}$ virus infection (3). Amongst these, gut flora serves a pivotal role in the pathogenesis of ALD and is closely associated with the liver in ALD via the gut-liver axis (4). Alcohol induces quantitative and qualitative alterations in the gut microbiota and increases gut permeability. This results in the translocation of endotoxins such as lipopolysaccharide (LPS) into the liver through the portal vein (5) and stimulates the release of pro-inflammatory mediators such as tumor necrosis factor (TNF)- $\alpha$ and interleukin (IL)- $1 \beta$ that ultimately contribute to liver damage in alcohol abuse patients (6).

Probiotics are live microorganisms that have demonstrated promise in ameliorating liver injury by the suppression of hepatic inflammation, regulation of intestinal microbiota and improvement of intestinal barrier integrity (7). Therefore, probiotics likely have therapeutic potential for ALD (8). Therapeutic strategies targeting the intestinal flora may be effective for ALD treatment (9). In addition, as the main source of energy for small intestinal cell metabolism, glutamine regulates intestinal barrier function by reducing its permeability, thereby preventing intestinal bacterial and endotoxin translocation (10). Glutamine also has research and application value in the treatment of diseases related to intestinal barrier damage (11). Most studies report the protective effect of glutamine on the intestinal barrier function based on the study of tight junctions; however, few studies have focused on the effect of the amino acid on intestinal microbiota $(12,13)$. 
It is therefore important to analyze intestinal flora to assess the effects of glutamine and probiotics, and clarify the mechanisms associated with ALD. The present study investigated alterations in the gut microbiota in response to chronic alcohol feeding followed by glutamine, Golden Bifido (probiotic mixture containing live Lactobacillus bulgaricus, Bifidobacterium and Streptococcus thermophilus) and Medilac- $\mathrm{S}^{\circledR}$ (probiotic mixturecontaining live Bacillus subtilis and Enterococcus faecium) treatment in ALD rats. The present results may help further understanding of glutamine and probiotics in ALD as well as the complexity of the interplay amongst probiotics, the gut flora, inflammation and ALD.

\section{Materials and methods}

Animals and treatments. A total of 60 male Sprague-Dawley rats were obtained from the Laboratory Animal Center of Fuzhou Wushi Animal Center (Fuzhou, China). Rats weighing $140 \pm 10 \mathrm{~g}$ and aged 8 weeks were housed at three per cage in an specific pathogen free animal room. Rats were housed in controlled conditions at a temperature of $20 \pm 2^{\circ} \mathrm{C}$, a relative humidity of $55 \pm 5 \%$ and 12 -h of light/dark cycle with free access to food and water.

For the experiment, following one week of adaptive feeding with a normal chow diet, the 60 rats were randomly divided into six groups as follows ( $n=10$; Fig. 1): i) C group: Normal chow diet and $1 \mathrm{ml} / \mathrm{kg} /$ day saline via intragastric administration for 20 weeks; ii) $\mathrm{M}$ group: Normal chow diet and intragastric ethanol ( $8 \mathrm{ml} / \mathrm{kg} /$ day; $56 \%$ ethanol $)$ on the first day, followed by a gradual increase to $18 \mathrm{ml} / \mathrm{kg} /$ day until the end of the experiment with a $2 \mathrm{ml} / \mathrm{kg} /$ day interval; iii) T group: Same method as the $\mathrm{M}$ group with intragastric administration of $1 \mathrm{ml} / \mathrm{kg} /$ day Golden Bifido suspension (total concentration, 500 mg/1 ml saline; cat. no. S1998004; Inner Mongolia Shuang Qi Pharmaceutical Co., Ltd) for the last 8 weeks; iv) G group: Same method as the M group with the intragastric administration of $1 \mathrm{ml} / \mathrm{kg} /$ day glutamine suspension (total concentration of $1 \mathrm{~g} / 1 \mathrm{ml}$ saline; cat. no. 62010836; Sinopharm Chemical Reagent Co., Ltd.) for the last 8 weeks; v) N group: Same method as the $\mathbf{M}$ group with the intragastric administration of $1 \mathrm{ml} / \mathrm{kg} /$ day Medilac-S ${ }^{\circledR}$ suspension (total concentration of $140 \mathrm{mg} / 1 \mathrm{ml}$ saline; cat. no. S20030087; Beijing Hanmei Pharmaceutical Co., Ltd.) for the last 8 weeks; and vi) the L group: Same method as the $M$ group with the intragastric administration of a Golden Bifido suspension $(500 \mathrm{mg} / \mathrm{kg} / \mathrm{day})$ and a glutamine suspension (140 $\mathrm{mg} / \mathrm{kg} /$ day) for the last 8 weeks. Doses of the treatment agents were chosen on the basis of previous studies $(14,15)$.

Subjects were weighed and then anesthetized using an intraperitoneal injection of $10 \%$ chloral hydrate $(300 \mathrm{mg} / \mathrm{kg})$ at the end of 20 weeks then rats were sacrificed by cervical dislocation. Blood, liver, intestinal tissues and feces were collected for subsequent biochemical analysis. All procedures were approved by the Institutional Animal Care and Use Committee of Fujian Medical University (registration number: 2015-CX-1).

Serum biochemical estimation. Blood samples were kept at room temperature for $1 \mathrm{~h}$ and then centrifuged at $1,500 \mathrm{x} \mathrm{g}$ for $15 \mathrm{~min}$ at $4^{\circ} \mathrm{C}$. The serum was stored at $-80^{\circ} \mathrm{C}$. Serum aspartate aminotransferase (AST), alanine aminotransferase (ALT) and triglyceride (TG) levels were measured using an automatic biochemical analyzer (Konelab 20; Thermo Fisher Scientific, Inc.). Tumor necrosis factor (TNF)- $\alpha$ (cat. no. SEA133Ra), interleukin (IL)-6 (cat. no. SEA079Ra), diamine oxidase (DAO) (cat. no. SEA656Ra), endotoxin (cat. no. CEB526Ge), occludin (cat. no. SEC228Ra) and D-lactate (cat. no. CEV643Ge) levels were detected by the relevant ELISA kits according to the manufacturer's instructions (All from Wuhan USCN Business Co., Ltd.). Each experiment was performed at least three times.

Pathologic evaluation. Each liver was fixed in $10 \%$ formalin solution for $36 \mathrm{~h}$ at $37^{\circ} \mathrm{C}$, embedded in paraffin, sectioned into $4 \mu \mathrm{m}$-thick slices and stained with hematoxylin for $10 \mathrm{~min}$ at $37^{\circ} \mathrm{C}$. Stained sections were imaged using a light microscope (Leica DM200; Leica Microsystems Ltd.) using a 40X objective lens and a color camera.

Gut microbiota analysis. Fresh feces (3-5 g) was obtained by a sterile swab, placed in an anaerobic dilution solution (4.5 g/l KH${ }_{2} \mathrm{PO}_{4} ; 6 \mathrm{~g} / 1 \mathrm{Na}_{2} \mathrm{HPO}_{4} ; 0.5 \mathrm{~g} / \mathrm{l} \mathrm{L-cysteine-HCl} ; 2 \mathrm{~g} / 1$ gelatin; $1 \mathrm{ml} / 1$ Tween-20) and analyzed by Hangzhou Jinghang Biotechnology Technology Co., Ltd. (Hangzhou, China) for $16 \mathrm{~S}$ rDNA sequencing.

Western blot analysis. A total of $0.1 \mathrm{~g}$ of intestinal tissue sample were ground, homogenized and then treated with radioimmunoprecipitation assay buffer (Thermo Fisher Scientific, Inc.). Protein content was determined by the bicinchoninic acid method. Equal amounts of protein $(30 \mu \mathrm{g})$ were separated via $10 \%$ SDS-PAGE and transferred to a polyvinylidene difluoride membrane. PVDF membranes were blocked with $5 \%$ fat-free milk powder diluted in TBS-T $(0.05 \%$ Tween 20$)$ at $37^{\circ} \mathrm{C}$ for $2 \mathrm{~h}$. The membranes were incubated with the primary antibodies against occludin (1:1,000; cat. no. ab167161) and $\beta$-actin (1:1,000; cat. no. ab8227; both Abcam, Cambridge, UK) at $4^{\circ} \mathrm{C}$ overnight. After rinsing three times with Tris-buffered saline and Polysorbate 20, the membranes were incubated with the corresponding horseradish peroxidase-labeled secondary antibody (1:1,000; cat. no. 31470; Thermo Fisher Scientific, Inc.) at $37^{\circ} \mathrm{C}$ for $2 \mathrm{~h}$ in the dark. Protein bands were detected using the Pierce ${ }^{\mathrm{TM}}$ ECL Western Blotting Substrate (cat. no. 32209; Thermo Fisher Scientific, Inc.) and Image lab software 3.0 (Bio-Rad Laboratories, Inc.). Relative protein expression levels were normalized to that of $\beta$-actin. Each experiment was performed at least three times.

Statistical analysis. Data are expressed as the mean \pm standard deviation. SPSS 16.0 (SPSS, Inc.) statistical software was used for statistical analysis. One-way ANOVA was used to compare differences amongst multiple groups followed by Dunnett's post hoc test. $\mathrm{P}<0.05$ was considered to indicate statistical significance.

\section{Results}

Glutamine and probiotic treatments elevate the body weight $(B W)$ of rats with ALD. As shown in Fig. 2, the BW of ALD 


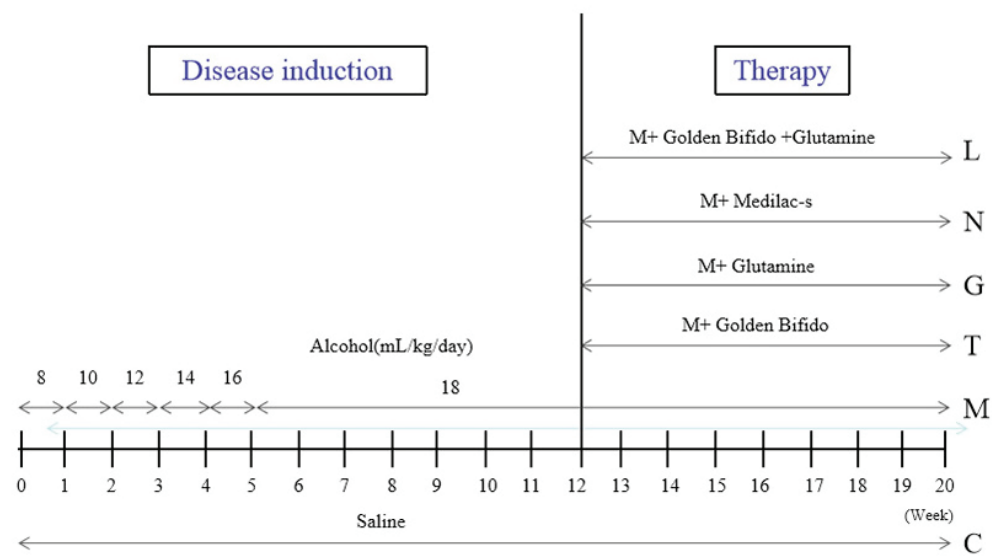

Figure 1. The study design and timeline. Schematic representation of the timeline for feeding rats with different concentrations of alcohol, Golden Bifido, glutamine, and Medilac-S ${ }^{\circledR}$. Treatments were as follows: i) $\mathrm{C}$ group: Normal chow diet and $1 \mathrm{ml}$ saline via intragastric administration for 20 weeks; ii) M group: Normal chow diet and intragastric ethanol $(8 \mathrm{ml} / \mathrm{kg} / \mathrm{day} ; 56 \%$ ethanol) on the first day, followed by a gradual increase to $18 \mathrm{ml} / \mathrm{kg} / \mathrm{day}$ until the end of the experiment with a $2 \mathrm{ml} / \mathrm{kg} /$ day interval; iii) T group: Same method as the M group with intragastric administration of $1 \mathrm{ml} / \mathrm{kg} / \mathrm{day}$ Golden Bifido suspension (total concentration of $500 \mathrm{mg} / 1 \mathrm{ml}$ saline) for the last 8 weeks; iv) G group: Same method as the M group with the intragastric administration of $1 \mathrm{ml} / \mathrm{kg} / \mathrm{day}$ glutamine suspension (total concentration of $1 \mathrm{~g} / 1 \mathrm{ml}$ saline) for the last 8 weeks; v) $\mathrm{N}$ group: Same method as the $\mathrm{M}$ group with the intragastric administration of $1 \mathrm{ml} / \mathrm{kg} /$ day Medilac-S ${ }^{\circledR}$ suspension (total concentration of $140 \mathrm{mg} / 1 \mathrm{ml}$ saline) for the last 8 weeks; and vi) the L group: Same method as the M group with the intragastric administration of a Golden Bifido suspension $(500 \mathrm{mg} / \mathrm{kg} /$ day $)$ and a glutamine suspension ( $140 \mathrm{mg} / \mathrm{kg} /$ day) for the last 8 weeks.

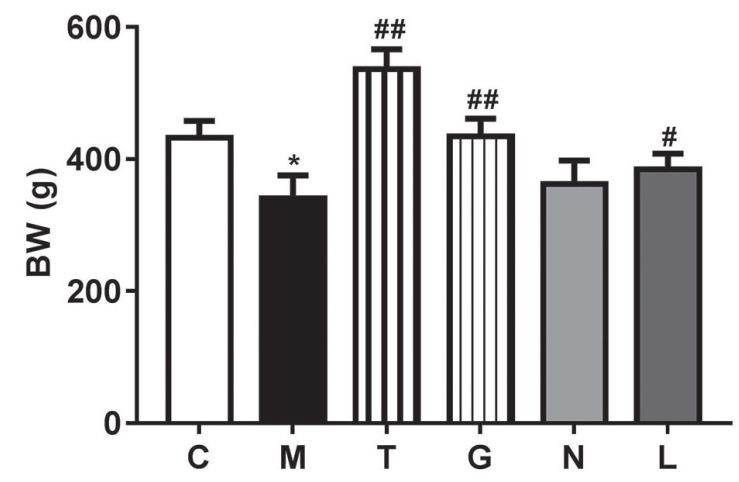

Figure 2. Final BW of ALD rats in each group after 20 weeks of treatment. ${ }^{*} \mathrm{P}<0.05$ vs. $\mathrm{C}$ group; ${ }^{*} \mathrm{P}<0.05$ and ${ }^{\# \#} \mathrm{P}<0.01$ vs. $\mathrm{M}$ group. $\mathrm{BW}$, body weight; ALD, alcoholic liver disease; C, control group; M, ALD group; T, Golden Bifid treatment group; G, Glutamine treatment group; N, Medilac- $\mathrm{S}^{\circledR}$ treatment group; L, Golden Bifido and glutamine treatment group.

rats was obviously lower than the control $(\mathrm{P}<0.05)$. However, the $\mathrm{BW}$ of rats in the $\mathrm{T}, \mathrm{G}$ and $\mathrm{L}$ groups was significantly higher compared with rats in the $\mathrm{M}$ group $(\mathrm{P}<0.01, \mathrm{P}<0.01$ and $\mathrm{P}<0.05$, respectively). There was no difference in body weight between the $\mathrm{N}$ and $\mathrm{M}$ groups $(\mathrm{P}>0.05)$.

Glutamine and probiotic treatments attenuate hepatic histopathological injury in rats with $A L D$. H\&E staining of rat livers demonstrated serious hepatic fatty changes, necrosis and inflammation in ALD rats. However, rat liver tissues from the T, G, N and $\mathrm{L}$ groups exhibited distinctly reduced alcohol-induced hepatic histopathological injury in ALD rats (Fig. 3).

Glutamine and probiotic treatments reduce serum ALT, TG and AST levels in rats with ALD. ALD rats had significantly higher ALT, TG and AST serum levels compared with the normal controls $(\mathrm{P}<0.01$; Table I). In contrast, serum AST,
Table I. AST, ALT and TG levels in each group.

\begin{tabular}{llcc}
\hline Group & ALT (U/l) & TG (mmol/l) & AST (U/l) \\
\hline C & $19.92 \pm 4.98$ & $0.74 \pm 0.03$ & $59.26 \pm 6.23$ \\
M & $43.59 \pm 0.61^{\mathrm{a}}$ & $1.95 \pm 0.06^{\mathrm{a}}$ & $65.38 \pm 2.24^{\mathrm{a}}$ \\
$\mathrm{T}$ & $36.66 \pm 1.12^{\mathrm{b}}$ & $1.62 \pm 0.36^{\mathrm{b}}$ & $52.94 \pm 2.92^{\mathrm{b}}$ \\
$\mathrm{G}$ & $34.56 \pm 4.05^{\mathrm{b}}$ & $1.52 \pm 0.04^{\mathrm{b}}$ & $47.62 \pm 4.56^{\mathrm{b}}$ \\
$\mathrm{N}$ & $35.42 \pm 3.53^{\mathrm{b}}$ & $1.47 \pm 0.04^{\mathrm{b}}$ & $49.62 \pm 1.22^{\mathrm{b}}$ \\
L & $23.38 \pm 4.91^{\mathrm{b}}$ & $0.90 \pm 0.14^{\mathrm{b}}$ & $37.61 \pm 3.37^{\mathrm{b}}$ \\
\hline
\end{tabular}

Data are reported as mean $\pm \mathrm{SD}, \mathrm{n}=10 .{ }^{\mathrm{a}} \mathrm{P}<0.01$ vs. $\mathrm{C}$ group; ${ }^{\mathrm{b}} \mathrm{P}<0.01$ vs. M group. ALT, alanine aminotransferase; TG, triglycerides; AST, aspartate transaminase. C, control group; M, ALD group; T, Golden Bifid treatment group; G, Glutamine treatment group; N, Medilac- $\mathrm{S}^{\circledR}$ treatment group; L, Golden Bifido and glutamine treatment group.

ALT, and TG levels in T, G, L and N groups were significantly lower compared with the ALD group $(\mathrm{P}<0.01$; Table I).

Glutamine and probiotic treatments decrease serum DAO, endotoxin and $D$-lactic acid levels in rats with $A L D$. As shown in Table II, the ALD rats had significantly higher serum DAO, endotoxin and D-lactate levels compared with the controls $(\mathrm{P}<0.01)$. Following 20 weeks of various glutamine and probiotic treatments, the serum DAO, endotoxin and D-lactic acid levels in the T, G, N and $\mathrm{L}$ groups were significantly decreased compared with the $\mathrm{M}$ group $(\mathrm{P}<0.01$; Table II).

Glutamine and probiotic treatments elevate occludin levels and reduce serum inflammatory factor levels in rats with $A L D$. After chronic alcohol feeding, the $\mathrm{M}$ group exhibited decreased occludin expression and elevated serum TNF- $\alpha$ and IL-6 levels compared with those in the $\mathrm{C}$ group $(\mathrm{P}<0.01$; Fig. 4; Table III). However, rats in the T, G, L, and N groups 

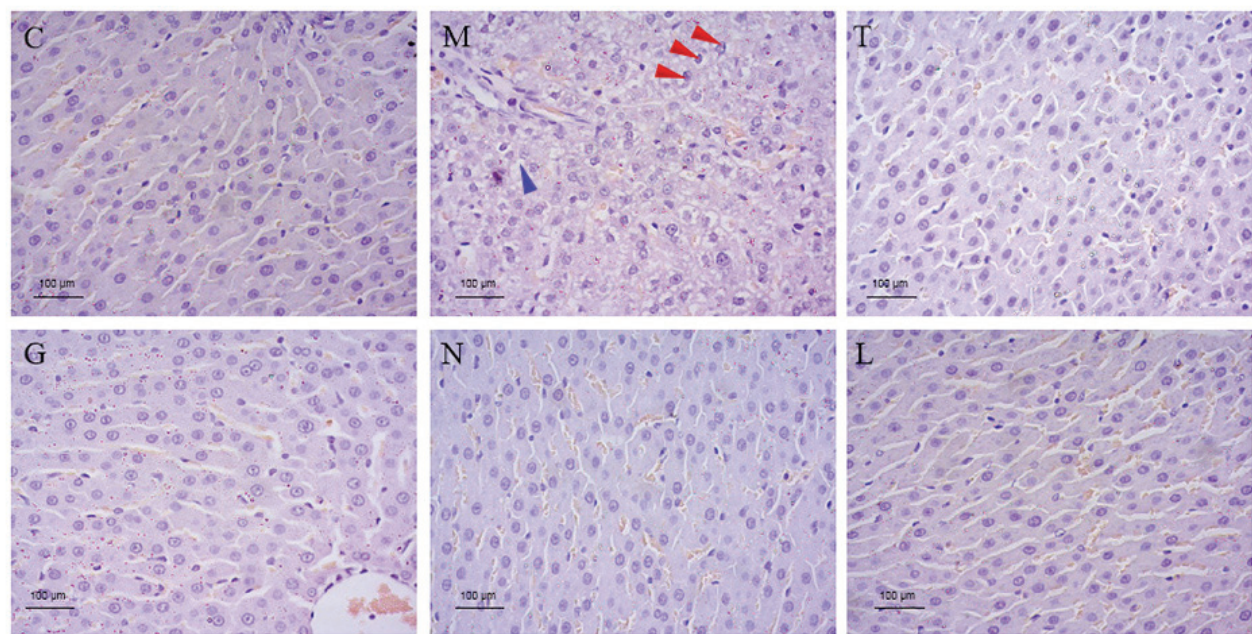

Figure 3. Hepatic histopathological analysis of ALD rats following various probiotic and glutamine treatments. Representative photomicrographs following hematoxylin and eosin staining to observe the morphology of livers from different groups. Red arrows represented necrosis and inflammation and blue arrow represented fatty change. Scale bar, $100 \mu \mathrm{m}$. ALD, alcoholic liver disease; C, control group; M, ALD group; T, Golden Bifid treatment group; G, Glutamine treatment group; N, Medilac- $\mathrm{S}^{\circledR}$ treatment group; L, Golden Bifido and glutamine treatment group.

Table II. Serum DAO, endotoxin and D-lactic acid levels in each group.

\begin{tabular}{lccc}
\hline Group & $\begin{array}{c}\text { DAO } \\
(\mathrm{mg} / \mathrm{ml})\end{array}$ & $\begin{array}{c}\text { Endotoxin } \\
(\mathrm{EU} / \mathrm{ml})\end{array}$ & $\begin{array}{c}\text { D-lactic } \\
\text { acid }(\mathrm{mg} / \mathrm{l})\end{array}$ \\
\hline $\mathrm{C}$ & $6.11 \pm 0.07$ & $0.66 \pm 0.018$ & $8.73 \pm 0.04$ \\
$\mathrm{M}$ & $7.88 \pm 1.10^{\mathrm{a}}$ & $0.86 \pm 0.05^{\mathrm{a}}$ & $15.46 \pm 0.31^{\mathrm{a}}$ \\
$\mathrm{T}$ & $6.51 \pm 0.59^{\mathrm{b}}$ & $0.76 \pm 0.01^{\mathrm{b}}$ & $13.47 \pm 0.13^{\mathrm{b}}$ \\
$\mathrm{G}$ & $6.72 \pm 0.19^{\mathrm{b}}$ & $0.74 \pm 0.02^{\mathrm{b}}$ & $13.86 \pm 0.07^{\mathrm{b}}$ \\
$\mathrm{N}$ & $6.54 \pm 0.40^{\mathrm{b}}$ & $0.77 \pm 0.004^{\mathrm{b}}$ & $14.20 \pm 0.17^{\mathrm{b}}$ \\
$\mathrm{L}$ & $6.34 \pm 1.21^{\mathrm{b}}$ & $0.70 \pm 0.01^{\mathrm{b}}$ & $10.43 \pm 0.15^{\mathrm{b}}$ \\
\hline
\end{tabular}

Data are reported as mean $\pm \mathrm{SD}, \mathrm{n}=10$. ${ }^{\mathrm{a}} \mathrm{P}<0.01$ vs. $\mathrm{C}$ group; ${ }^{\mathrm{b}} \mathrm{P}<0.01$ vs. $\mathrm{M}$ group. DAO, diamine oxidase. C, control group; $\mathrm{M}, \mathrm{ALD}$ group; T, Golden Bifid treatment group; G, Glutamine treatment group; N, Medilac- $S^{\circledR}$ treatment group; L, Golden Bifido and glutamine treatment group.

Table III. IL6, occludin and TNF- $\alpha$ levels in each group.

\begin{tabular}{llcc}
\hline Group & IL-6 (ng/l) & Occludin (ng/l) & $\begin{array}{c}\text { TNF- } \alpha \\
(\mathrm{ng} / \mathrm{l})\end{array}$ \\
\hline $\mathrm{C}$ & $3.47 \pm 0.07$ & $48.22 \pm 5.58$ & $1.92 \pm 0.12$ \\
$\mathrm{M}$ & $5.53 \pm 0.13^{\mathrm{a}}$ & $35.24 \pm 9.55^{\mathrm{a}}$ & $3.70 \pm 0.14^{\mathrm{a}}$ \\
$\mathrm{T}$ & $4.82 \pm 0.17^{\mathrm{b}}$ & $39.03 \pm 3.23^{\mathrm{b}}$ & $3.07 \pm 0.20^{\mathrm{b}}$ \\
$\mathrm{G}$ & $4.64 \pm 0.39^{\mathrm{b}}$ & $40.51 \pm 1.32^{\mathrm{b}}$ & $2.74 \pm 0.10^{\mathrm{b}}$ \\
$\mathrm{N}$ & $4.91 \pm 0.37^{\mathrm{b}}$ & $41.07 \pm 8.39^{\mathrm{b}}$ & $2.69 \pm 0.14^{\mathrm{b}}$ \\
L & $3.86 \pm 0.48^{\mathrm{b}}$ & $41.76 \pm 4.55^{\mathrm{b}}$ & $2.22 \pm 0.12^{\mathrm{b}}$ \\
\hline
\end{tabular}

Data are reported as mean $\pm \mathrm{SD}, \mathrm{n}=10 .{ }^{\mathrm{a}} \mathrm{P}<0.01$ vs. $\mathrm{C}$ group; ${ }^{\mathrm{b}} \mathrm{P}<0.01$ vs. $\mathrm{M}$ group. IL-6, interleukin-6; TNF- $\alpha$, tumor necrosis factor. $\mathrm{C}$, control group; M, ALD group; T, Golden Bifid treatment group; $\mathrm{G}$, Glutamine treatment group; N, Medilac-S ${ }^{\circledR}$ treatment group; $\mathrm{L}$, Golden Bifido and glutamine treatment group.

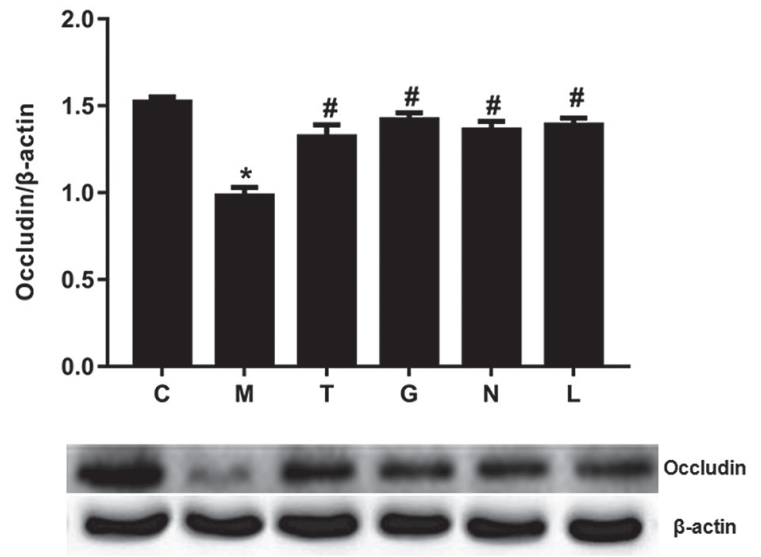

Figure 4. Western blot analysis of the occludin protein expression level in intestinal tissue of ALD rats following various probiotic and glutamine treatments. ${ }^{*} \mathrm{P}<0.05$ vs. $\mathrm{C}$ group; ${ }^{\#} \mathrm{P}<0.05$ vs. $\mathrm{M}$ group. $\mathrm{C}$, control group; $\mathrm{M}, \mathrm{ALD}$ group; T, Golden Bifid treatment group; G, Glutamine treatment group; N, Medilac- $S^{\circledR}$ treatment group; L, Golden Bifido and glutamine treatment group.

demonstrated significantly reduced TNF- $\alpha$ and IL-6 levels $(\mathrm{P}<0.01$; Table III) and increased occludin protein expression $(\mathrm{P}<0.05$; Fig. 4) compared with the M group.

Glutamine and probiotic treatments modulate gut microbiota in rats with $A L D$. At the phylum level, the abundance of Firmicutes was notably decreased in the ALD group compared with the healthy controls $(\mathrm{P}<0.05$; Fig. 5), whilst the proportion of Bacteroidetes was not significantly different in ALD and control rats. The proportion of Proteobacteria $(\mathrm{P}<0.05$; Fig. 5) and Actinobacteria ( $\mathrm{P}<0.01$; Fig. 5) in the ALD group was significantly higher compared with the control group. However, treatment with glutamine and probiotics increased the abundance of Firmicutes $(\mathrm{P}<0.05)$ and decreased the abundance of Proteobacteria $(\mathrm{P}<0.05)$ and Actinobacteria $(\mathrm{P}<0.05$ $\mathrm{P}<0.01, \mathrm{P}<0.05$ and $\mathrm{P}<0.01$, respectively) in $\mathrm{T}, \mathrm{G}, \mathrm{L}$, and $\mathrm{N}$ groups compared with the $\mathrm{M}$ groups (Fig. 5). 

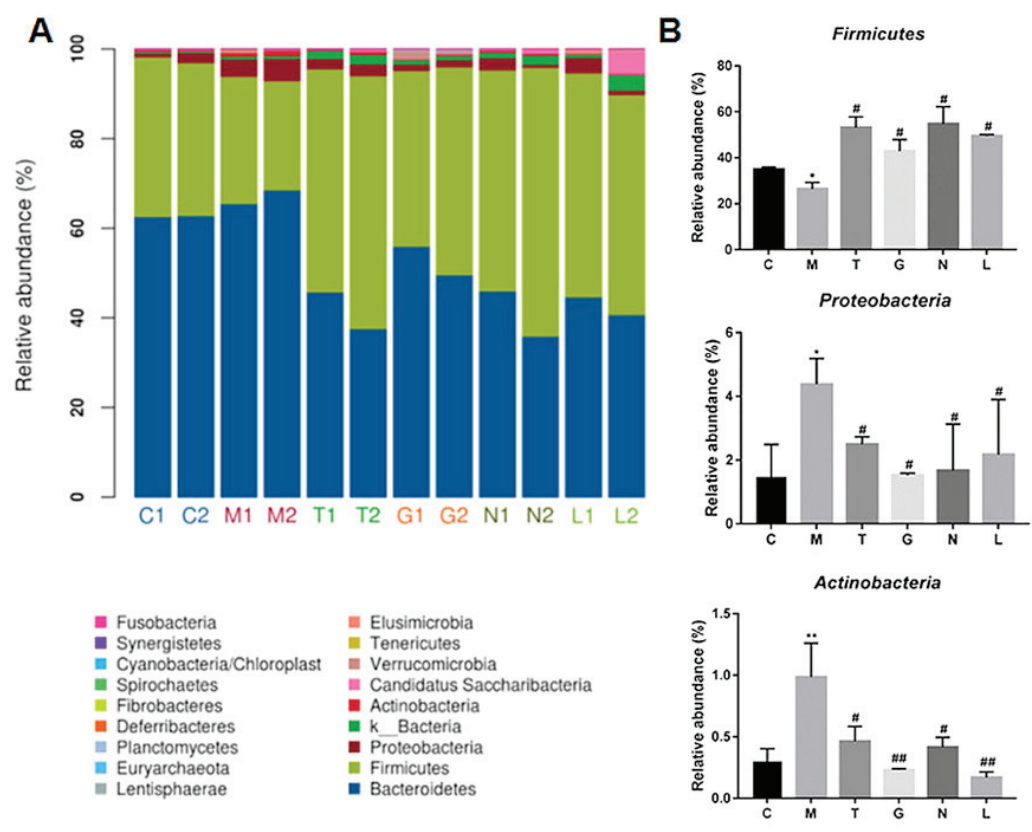

Figure 5. Bacterial community structures at the phylum level. (A) The abundance of each bacteria genus is presented as the percentage of the total effective bacterial sequences in the sample. (B) The relative abundance of Firmicutes, Proteobacteria and Actinobacteria in each group. ${ }^{*} \mathrm{P}<0.05$ and ${ }^{* *} \mathrm{P}<0.05$ vs. $\mathrm{C}$ group; ${ }^{\#} \mathrm{P}<0.05$ and ${ }^{\# \#} \mathrm{P}<0.01$ vs. $\mathrm{M}$ group. $\mathrm{C}$, control group; $\mathrm{M}$, ALD group; T, Golden Bifid treatment group; G, Glutamine treatment group; N, Medilac-S ${ }^{\circledR}$ treatment group; L, Golden Bifido and glutamine treatment group.
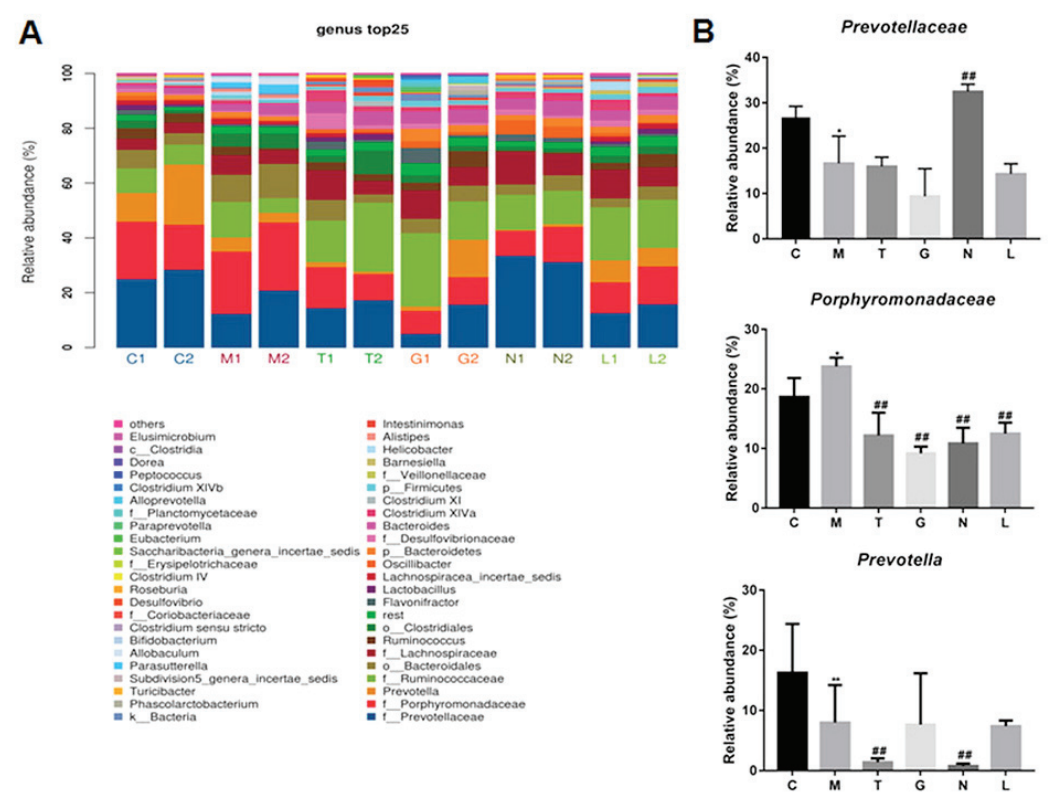

Figure 6. Bacterial community structures at the genus level. (A) The abundance of each bacteria genus is presented as the percentage of the total effective bacterial sequences in the sample. (B) The relative abundance of Prevotellaceae, Porphyromonadaceae and Prevotella in each group. ${ }^{*} \mathrm{P}<0.05$ and ${ }^{* * *} \mathrm{P}<0.05$ vs. C group; ${ }^{\# \#} \mathrm{P}<0.01$ vs. M group. C, control group; M, ALD group; T, Golden Bifid treatment group; G, Glutamine treatment group; $\mathrm{N}$, Medilac- ${ }^{\circledR}$ treatment group; L, Golden Bifido and glutamine treatment group.

At the genus level, Prevotellaceae was the most prevalent genus in healthy controls and significantly decreased in the ALD group $(\mathrm{P}<0.05$; Fig. 6$)$. Moreover, the proportion of Prevotellaceae in the $\mathrm{N}$ group was significantly higher compared with the ALD group $(\mathrm{P}<0.01$; Fig. 6). Porphyromonadaceae was the second most prevalent genus in the control group. Alcohol infusion induced a significant increase in Porphyromonadaceae in comparison with the control group ( $\mathrm{P}<0.05$; Fig. 6). However, Porphyromonadaceae abundance was reduced in T, G, L, and N groups when compared with the ALD group ( $\mathrm{P}<0.01$; Fig. 6). Prevotella was the third most prevalent genus in healthy controls, whereas their relative abundance was reduced by alcohol treatment ( $<<0.01$; Fig. 6). Following glutamine and probiotic treatments, Prevotella abundance in the $\mathrm{T}$ and $\mathrm{N}$ groups were significantly decreased compared with the $\mathrm{M}$ group ( $\mathrm{P}<0.01$; Fig. 6$)$. 


\section{Discussion}

Chronic ethanol feeding destroys the integrity of the intestinal barrier and disturbs the gut microbiota $(16,17)$. These alterations increase the translocation of endotoxins such as LPS from the intestinal lumen to the portal blood (18). LPS then binds to toll like receptor 4 on the surface of hepatic Kupffer cells to produce inflammatory cytokines which ultimately results in ALD (19). Therefore, the restoration of intestinal homeostasis could be a potential therapy for ALD. Many studies have reported that alcohol exposure decreased final BW in chronic ALD $(20,21)$. The present study identified that BW was lower in the ALD group compared with the control group at 20 weeks. However, treatments with Golden Bifido and glutamine attenuated the reduction in BW induced by alcohol feeding.

Epithelial tight junctions are the primary component of the intestinal mucosal barrier (12). Occludin is an integral membrane protein localized at tight junctions (22) that has an important role in the maintenance of the basic structure and function of tight junctions. Chaudhry et al (23) reported that chronic alcohol consumption caused the redistribution of occludin from the intercellular junctions of the colonic epithelium and disrupted colonic epithelial tight junctions and intestinal epithelial barrier function; however, Glutamine supplementation protected tight junctions in the colonic epithelium of mice fed with alcohol and thus maintained intestinal epithelial barrier function. Moreover, serum ALT and AST levels can be used to indicate liver injury (24). D-Lactate, endotoxin and DAO levels reflect the severity of intestinal mucosa injury (25). Li et al (26) identified that an increase in these parameters occurred in rats that underwent chronic alcohol feeding. In the present study, abnormally decreased occludin levels and abnormally elevated plasma ALT, AST, TG, DAO, endotoxin and D-lactate levels were detected in the ALD group, indicating alcohol-induced liver injury and intestinal mucosa injury. Significant changes in these indexes in the sera of rats in all intervention groups revealed that probiotics and glutamine alleviated liver damage and intestinal mucosa injury caused by chronic alcohol use. Moreover, various inflammatory cytokines, such as TNF- $\alpha$ and IL-6, have been reported to be involved in the occurrence and development of ALD $(27,28)$. Previous studies have shown that glutamine and probiotics reduce inflammation, promote the secretion of anti-inflammatory cytokines and maintain intestinal barrier functions $(29,30)$. The present results demonstrated that probiotic and glutamine treatments reduced the abnormally elevated serum IL- 6 and TNF- $\alpha$ levels following chronic ethanol consumption, demonstrating that probiotics and glutamine likely alleviated hepatic inflammation via the suppression of inflammatory cytokines. Moreover, liver injury induced by alcohol at the histopathological level was completely reversed after probiotic and glutamine treatments, indicating the protective effect of probiotics and glutamine on liver damage.

Alcohol-induced intestinal dysbiosis is involved in the pathogenesis of $\operatorname{ALD}(4,31)$. In the present study, at the phylum level, Firmicutes was the most dominant phyla in healthy controls, which was consistent with previous studies (32).
The present results demonstrated decreased Firmicutes and higher Actinobacteria and Proteobacteria abundance at the phylum level in the ALD group, which were in agreement with the results obtained by Bull-Otterson et al (33). At the genus level, increased Porphyromonadaceae and decreased Prevotellaceae and Prevotella abundance were observed in ALD mice compared with the controls. The increase in the abundance of Porphyromonadaceae, elevated plasma endotoxin levels and hepatic inflammation are strongly associated with complications in chronic liver disease (34).

Previous studies have demonstrated that probiotics ameliorate alcohol-induced gut dysbiosis and prevent alcoholic liver injury $(7,35)$. In the present study, probiotics and glutamine notably elevated the abundance of Firmicutes and reduced Actinobacteria, Proteobacteria and Porphyromonadaceae abundance following continuous alcohol consumption, indicating that probiotic and glutamine treatments may attenuate gut dysbiosis.

In conclusion, the present study demonstrated that probiotic and glutamine treatments ameliorated ALD in rats via the suppression of inflammation and the regulation of intestinal microbiota. Results suggested that these interventions may potentially serve as inexpensive therapies for the prevention and treatment of ALD.

\section{Acknowledgements}

Not applicable.

\section{Funding}

The present study study was supported by Fujian Medical Innovation Foundation (grant no. 2015-CX-1).

\section{Availability of data and materials}

All data generated or analyzed during the present study are included in this published article.

\section{Authors' contributions}

ZL and YZ designed the study; HH, ZL and YZ performed the experiments; $\mathrm{HH}$ and XL collected the data; $\mathrm{HH}, \mathrm{XL}$ and $\mathrm{YZ}$ analyzed the data; and $\mathrm{YZ}$ and $\mathrm{XL}$ prepared the manuscript. All authors read and approved the final manuscript.

\section{Ethics approval and consent to participate}

All procedures were approved by the Institutional Animal Care and Use Committee of Fujian Medical University (registration number: 2015-CX-1).

\section{Patient consent for publication}

Not applicable.

\section{Competing interests}

The authors declare that they have no competing interests. 


\section{References}

1. Singal AK, Bataller R, Ahn J, Kamath PS and Shah VH: ACG clinical guideline: Alcoholic liver disease. Am J Gastroenterol 113: 175-194, 2018

2. Beier JI and Mcclain CJ: Mechanisms and cell signaling in alcoholic liver disease. Biol Chem 391: 1249-1264, 2010.

3. O'Shea RS, Dasarathy S and Mccullough AJ: Alcoholic liver disease. Hepatology 51: 227-229, 1995.

4. Szabo G: Gut-liver axis in alcoholic liverdisease. Gastroenterology 148: 30-36, 2015

5. Bode $\mathrm{C}$ and Bode JC: Effect of alcohol consumption on the gut Best Pract Res Clin Gastroenterol 17: 575-592, 2003.

6. Lucey MR, Mathurin P and Morgan TR: Alcoholic hepatitis. N Engl J Med 360: 2758-2769, 2009.

7. Malaguarnera G, Giordano M, Nunnari G, Bertino G and Malaguarnera M: Gut microbiota in alcoholic liver disease: Pathogenetic role and therapeutic perspectives. World J Gastroenterol 20: 16639-16648, 2014.

8. Komatsuzaki Nand Shima J: Effects of live lactobacillus paracasei on plasma lipid concentration in rats fed an ethanol-containing diet. Biosci Biotechnol Biochem 76: 232-237, 2012.

9. Sung H, Kim SW,Hong M and Suk KT: Microbiota-based treatments in alcoholic liver disease. World J Gastroenterol 22: 6673-6682, 2016.

10. White JS, Hoper M, Parks RW, Clements WD and Diamond T: Glutamine improves intestinal barrier function in experimental biliary obstruction. Eur Surg Res 37: 342-347, 2005.

11. dos Santos Rd, Viana ML, Generoso SV, Arantes RE, Davisson Correia MI and Cardoso VN: Glutamine supplementation decreases intestinal permeability and preserves gut mucosa integrity in an experimental mouse model. JPEN J Parenter Enteral Nutr 34: 408-413, 2010.

12. Li N, Lewis P, Samuelson D, Liboni K and Neu J: Glutamine regulates Caco-2 cell tight junction proteins. Am J Physiol Gastrointest Liver Physiol 287: G726-G733, 2004.

13. Li N and Neu J: Glutamine deprivation alters intestinal tight junctions via a PI3-K/Akt mediated pathway in Caco-2 cells. J Nutr 139: 710-714, 2009.

14. Sellmann C, Baumann A, Brandt A, Jin CJ, Nier A and Bergheim I: Oral supplementation of glutamine attenuates the progression of nonalcoholic steatohepatitis in $\mathrm{C} 57 \mathrm{BL} / 6 \mathrm{~J}$ mice. J Nutr 147: 2041-2049, 2017.

15. Huang H, Zeng Y, Lin H, Lin XY and Lin ZH: Effects of fecal microbiota transplantation and joint application of probiotics on rats with alcoholic liver disease. Int J Clin Exp Med 11: 12368-12374, 2018.

16. Hartmann P, Chen WC and Schnabl B: The intestinal microbiome and the leaky gut as therapeutic targets in alcoholic liver disease. Front Physiol 3: 402, 2012.

17. Addolorato G, Montalto M, Capristo E, Certo M, Fedeli G, Gentiloni N, Stefanini GF and Gasbarrini G: Influence of alcohol on gastrointestinal motility: Lactulose breath hydrogen testing in orocecal transit time in chronic alcoholics, social drinkers and teetotaler subjects. Hepatogastroenterology 44: 1076-1081, 1997.

18. Wang L, Llorente C, Hartmann P, Yang AM, Chen P and Schnabl B Methods to determine intestinal permeability and bacterial translocation during liver disease. J Immunol Methods 421: 44-53, 2015.

19. Lieber CS: Hepatic, metabolic and toxic effects of ethanol: 1991 update. Alcohol Clin Exp Res 15: 573-592, 1991.

20. Gao B, Xu MJ, Bertola A, Wang H, Zhou Z and Liangpunsakul S: Animal models of alcoholic liver disease: Pathogenesis and clinical relevance. Gene Expr 17: 173-186, 2017.
21. Tian F, Chi F, Wang G, Liu X, Zhang Q, Chen Y, Zhang H and Chen W: Lactobacillus rhamnosus CCFM1107 treatment ameliorates alcohol-induced liver injury in a mouse model of chronic alcohol feeding. J Microbiol 53: 856-863, 2015.

22. Furuse M, Hirase T, Itoh M, Nagafuchi A, Yonemura S, Tsukita S and Tsukita S: Occludin: A novel integral membrane protein localizing at tight junctions. J Cell Biol 123: 1777-1788, 1993

23. Chaudhry KK, Shukla PK, Mir H, Manda B, Gangwar R, Yadav N, McMullen M, Nagy LE and Rao R: Glutamine supplementation attenuates ethanol-induced disruption of apical junctional complexes in colonic epithelium and ameliorates gut barrier dysfunction and fatty liver in mice. J Nutr Biochem 27: $16-26,2016$

24. van Beek JH, de Moor MH, de Geus EJ, Lubke GH, Vink JM, Willemsen G and Boomsma DI: The genetic architecture of liver enzyme levels: GGT, ALT and AST. Behav Genet 43: 329-339, 2013.

25. Ruan P, Gong ZJ and Zhang QR: Changes of plasma D(-)-lactate, diamine oxidase and endotoxin in patients with liver cirrhosis. Hepatobiliary Pancreat Dis Int 3: 58-61, 2004.

26. Li H, Qiu P, Wang J, Niu C and Pan S: Effects of compound Ginkgo biloba on intestinal permeability in rats with alcohol-induced liver injury. Food Funct 6: 470-478, 2015.

27. Mcclain C, Hill D, Schmidt J and Diehl AM: Cytokines and alcoholic liver disease. Semin Liver Dis 13: 170-182, 1993.

28. Ciećko-Michalska I, Szczepanek M, Cibor D, Owczarek D, Skulina D, Szczepański W and Michalski M: Serum cytokine concentration as prognostic factor in patients with alcoholic liver disease. Przegl Lek 63: 249-252, 2006 (In Polish).

29. Gong ZY, Yuan ZQ, Dong ZW and Peng YZ: Glutamine with probiotics attenuates intestinal inflammation and oxidative stress in a rat burn injury model through altered iNOS gene aberrant methylation. Am J Transl Res 9: 2535-2547, 2017.

30. Hastings CN, Sheridan H, Pariante CM and Mondelli V: Does diet matter? The use of polyunsaturated fatty acids (PUFAs) and other dietary supplements in inflammation-associated depression. Curr Top Behav Neurosci 31: 321-338, 2016.

31. Cresci GA: The gut microbiome: A new frontier for alcohol investigation. Alcohol Clin Exp Res 39: 947-949, 2015.

32. Mutlu EA, Gillevet PM, Rangwala H, Sikaroodi M, Naqvi A, Engen PA, Kwasny M, Lau CK and Keshavarzian A: Colonic microbiome is altered in alcoholism. Am J Physiol Gastrointest Liver Physiol 302: G966-G978, 2012.

33. Bullotterson L, Feng W, Kirpich I, Wang Y, Xiang Q, Liu Y, Gobejishvili L, Joshi-Barve S, Ayvaz T, Petrosino J, et al: Metagenomic analyses of alcohol induced pathogenic alterations in the intestinal microbiome and the effect of lactobacillus rhamnosus GG treatment. PLoS One 8: e53028, 2013.

34. Bajaj JS, Ridlon JM, Hylemon PB, Thacker LR, Heuman DM, Smith S, Sikaroodi M and Gillevet PM: Linkage of gut microbiome with cognition in hepatic encephalopathy. Am J Physiol Gastrointest Liver Physiol 302: G168-G175, 2012.

35. Vassallo G, Mirijello A, Ferrulli A, Antonelli M, Landolfi R, Gasbarrini A and Addolorato G: Review article: Alcohol and gut microbiota-the possible role of gut microbiota modulation in the treatment of alcoholic liver disease. Aliment Pharmacol Ther 41: 917-927, 2015.

This work is licensed under a Creative Commons Attribution-NonCommercial-NoDerivatives 4.0 International (CC BY-NC-ND 4.0) License. 